\title{
Influência das cargas selecionadas na determinação da potência crítica determinada no ergômetro de braço em dois modelos lineares
}

\author{
João Francisco Francelin Calis e Benedito Sérgio Denadai
}

Laboratório de Avaliação da Performance Humana, Departamento de Educação Física, Unesp - Rio Claro, SP

\section{RESUMO}

O objetivo deste estudo foi verificar o efeito da seleção das cargas e do modelo utilizado para a determinação da PC no ergômetro de braço. Participaram do estudo oito voluntários do sexo masculino, que praticavam atividade física regularmente e eram aparentemente saudáveis. Os sujeitos realizaram quatro testes com cargas constantes mantidas até a exaustão voluntária no ergômetro de braço UBE 2462-Cybex. As cargas foram individualmente selecionadas para induzir a fadiga entre 1 e 15 minutos. Para cada sujeito, a determinação da PC foi realizada através de dois modelos lineares: potência-1/tempo e trabalho-tempo. Em cada um dos modelos, foram utilizadas todas as potências (1), as três maiores (2) e as três menores (3). As PC encontradas no modelo potência-1/ tempo e trabalho-tempo para a condição $3(177,5 \pm 29,5 ; 173,9 \pm 33,3$, respectivamente) foram significantemente menores do que as da condição 2 $(190,5 \pm 23,2 ; 183,4 \pm 22,3$, respectivamente), não existindo diferenças destas com as da condição 1 (184,2 \pm 25,4; $176,4 \pm 28,8$, respectivamente). As PC determinadas no modelo potência-1/tempo para as condições 1 e 2 foram significantemente maiores do que as determinadas no modelo trabalho-tempo, não existindo diferenças para a condição 3. Pode-se concluir que as cargas selecionadas e o modelo utilizado interferem na determinação da PC encontrada no ergômetro de braço, podendo interferir no tempo de exaustão durante o exercício submáximo realizado em cargas relativas a este índice.

Palavras-chave: Potência crítica. Modelo linear. Tempo de exaustão. Ergômetro de braço.

Endereço para correspondência:

B.S. Denadai

Departamento de Educação Física

Instituto de Biociências

Av. 24A, 1.515 - Bela Vista

13506-900 - Rio Claro, SP - Brasil

E-mail: bdenadai@rc.unesp.br

Rev Bras Med Esporte - Vol. 6, N 1 - Jan/Fev, 2000

\section{ABSTRACT}

Effect of selected loads on the critical power determination in the arm ergometer by two linear models

The aim of this study was to verify the effect of selected loads and the model used to determine CP in the arm ergometer. Eight active males apparently healthy participated in the study. The subjects performed 4 tests with constant loads maintained until the voluntary exhaustion in the arm ergometer (UBE 2462-Cybex). The loads were individually selected to induce fatigue between 1 and 15 minutes. For each subject, the determination of $C P$ was accomplished by two linear models: power-1/time and work-time. In each model, the CP was calculated using: all power outputs (1), the three highest (2), and the three lowest outputs (3). The CP in the power-1/time and work-time models for the condition 3 (177.5 $\pm 29.5 ; 173.9 \pm 33.3$, respectively) were lower than for the condition 2 (190.5 \pm 23.2 ; $183.4 \pm 22.3$, respectively), no difference existing between condition 2 and 1 (184.2 $\pm 25.4 ; 176.4 \pm 28.8$, respectively). The CP determined in the power-1/time model for conditions 1 and 2 were higher than in the work-time model, no difference existing for condition 3. It can be concluded that the selected loads and the model used interfere in the determination of CP during arm crank exercise, and can modify the time to exhaustion during submaximal exercise performed in relative loads to $C P$.

Key words: Critical power. Linear model. Time to exhaustion. Arm ergometer.

\section{INTRODUÇÃO}

O conceito de potência crítica (PC) foi inicialmente proposto por Monod e Scherrer ${ }^{1}$ para grupos musculares sinergistas, sendo posteriormente estendido por Moritani et $a l .{ }^{2}$ para grandes grupos musculares (ciclismo). A PC é um conceito teórico que pressupõe a existência de uma potência máxima de exercício, que pode ser mantida indefinida- 
mente. $\mathrm{O}$ conceito é baseado na relação hiperbólica entre a potência realizada $\left(\mathrm{W}_{\lim }\right)$ e seu respectivo tempo de exaustão $\left(t_{\text {lim }}\right)$. Nesta relação, pode ser determinada a capacidade de trabalho anaeróbio (CTAn), já tendo sido verificadas correlações moderadas deste índice com a performance anaeróbia ${ }^{3,4}$ e a PC propriamente dita, que apresenta elevada correlação com a performance aeróbia ${ }^{5,6}$. Três diferentes modelos têm sido utilizados para a determinação desses índices: 1) modelo hiperbólico da relação potência-tempo, em que $\left.\mathrm{t}_{\lim }=\mathrm{CTAn} /\left(\mathrm{W}_{\lim }-\mathrm{PC}\right) ; 2\right)$ modelo linear potência-1/tempo, em que $\mathrm{W}_{\lim }=\left(\mathrm{CTAn} / \mathrm{t}_{\lim }\right)+\mathrm{PC}$ e; 3$)$ modelo trabalho-tempo, em que Trabalho $=\left(\right.$ PC. $\left.t_{\text {lim }}\right)+$ CTAn.

A validade da PC como índice de capacidade aeróbia parece ser bastante elevada, pois vários estudos utilizando diferentes modelos para sua determinação têm encontrado altas correlações entre a potência crítica e o limiar anaeróbio e/ou a performance aeróbia, independentemente do tipo de exercício, sexo e faixa etária ${ }^{5-7}$.

Entretanto, alguns estudos verificaram que a intensidade de exercício correspondente à $\mathrm{PC}$ pode ser significantemente maior que a do limiar anaeróbio ${ }^{9}$. Além disso, temse verificado também que o tempo de exaustão na PC tem variado bastante, alguns estudos encontrando tempos relativamente reduzidos e/ou altas concentrações de lactato ao final do exercício ${ }^{9-11}$. Em razão disso, a validade da utilização da PC para a prescrição da intensidade de treinamento aeróbio tem sido questionada.

Esse comportamento pode ter ocorrido em função das cargas selecionadas para a identificação da PC. Bishop et $a l .{ }^{12}$ verificaram, para o exercício na bicicleta ergométrica, que independente do modelo utilizado para a identificação da PC (hiperbólico ou linear), o emprego de cargas mais elevadas e, conseqüentemente, com menores tempos de exaustão, produziu valores de PC significantemente maiores do que quando se utilizaram cargas de menor potência. Esta influência das cargas selecionadas para induzir a exaustão no cálculo da PC também tem sido encontrada em outros estudos que utilizaram a bicicleta ergométrica como modelo de exercício ${ }^{13}$.

Por outro lado, não foram encontrados estudos que tenham analisado essa influência no exercício realizado no ergômetro de braço, que utiliza uma massa muscular significantemente menor do que na bicicleta ergométrica. Essas informações são muito interessantes, já que o ergômetro de braço tem sido utilizado para a avaliação e o treinamento de populações especiais e atletas. Desse modo, o objetivo deste estudo foi verificar se as cargas selecionadas e o modelo (potência-1/tempo e trabalho-tempo) utilizado interferem no cálculo da PC e da CTAn determinadas no ergômetro de braço.

\section{MATERIAL E MÉTODOS}

\section{Sujeitos}

Participaram do estudo oito voluntários do sexo masculino, que praticavam atividade física regularmente e eram aparentemente saudáveis. Após a explicação de todos os procedimentos experimentais, os indivíduos assinaram um termo de consentimento. As características dos participantes encontram-se na tabela 1.

\section{Testes}

Os indivíduos participaram de três sessões experimentais, separadas por no mínimo 24 horas, sendo a duração total de 5-7 dias.

Na primeira sessão, além da assinatura do termo de consentimento, os indivíduos foram familiarizados com os procedimentos e o ergômetro de braço (UBE 2462 - Cybex).

Nas duas sessões seguintes, os indivíduos se exercitaram até a exaustão voluntária, em duas intensidades (potências) diferentes, com um intervalo mínimo de 30 minutos entre cada uma. Em cada teste, os sujeitos pedalaram a 90rpm, em potências situadas entre 150 e 300 watts, de acordo com a sua capacidade funcional. Cada potência foi selecionada para determinar a exaustão entre 1 e 15 minutos (tabela 2).

A ordem de execução de cada intensidade foi aleatória. Todos os testes foram realizados nos mesmo horário do dia e a temperatura ambiente variou entre 25 e $28^{\circ} \mathrm{C}$.

\section{Cálculo da PC e CTAn}

Para cada sujeito, a determinação da PC e da CTAn foi realizada através de dois modelos lineares: potência-1/tempo e trabalho-tempo. Em cada um dos modelos, foram uti-

\begin{tabular}{|c|c|c|c|c|c|c|c|}
\hline \multicolumn{8}{|c|}{$\begin{array}{c}\text { TABELA } 1 \\
\text { Características dos indivíduos }(\mathrm{N}=8)\end{array}$} \\
\hline \multicolumn{2}{|c|}{$\begin{array}{l}\text { Idade } \\
\text { (anos) }\end{array}$} & \multicolumn{2}{|c|}{$\begin{array}{l}\text { Peso } \\
\text { (kg) }\end{array}$} & \multicolumn{2}{|c|}{$\begin{array}{l}\text { Altura } \\
\text { (cm) }\end{array}$} & \multicolumn{2}{|c|}{$\begin{array}{l}\text { Percentual de } \\
\text { gordura }(\%)\end{array}$} \\
\hline \multicolumn{2}{|c|}{$26,2 \pm 4,0$} & \multicolumn{2}{|c|}{$73,5 \pm 5,8$} & \multicolumn{2}{|c|}{$172,4 \pm 1,6$} & \multicolumn{2}{|c|}{$13,3 \pm 6,8$} \\
\hline \multicolumn{8}{|c|}{$\begin{array}{c}\text { TABELA } 2 \\
\text { Valores médios } \pm \text { DP da potência }\left(W_{1 i m}\right) \text { e do seu } \\
\text { respectivo tempo de exaustão }\left(t_{1 i m}\right) \text { em cada teste } \\
\text { para a determinação da potência crítica e da } \\
\text { capacidade de trabalho anaeróbio }(N=8)\end{array}$} \\
\hline \multicolumn{3}{|c|}{ Teste 1} & \multicolumn{2}{|c|}{ Teste 2} & \multicolumn{2}{|c|}{ Teste 3} & Teste 4 \\
\hline$W_{\text {lim }}(W)$ & \multicolumn{2}{|c|}{$275,0 \pm 21,4$} & \multicolumn{2}{|c|}{$237,5 \pm 19,2$} & \multicolumn{2}{|c|}{$207,8 \pm 15,5$} & $189 \pm 14,8$ \\
\hline$t_{\text {lim }}(\min )$ & \multicolumn{2}{|c|}{$1,5 \pm 0,4$} & \multicolumn{2}{|c|}{$2,9 \pm 1,1$} & \multicolumn{2}{|c|}{$6,0 \pm 1,0$} & $12,1 \pm 4,6$ \\
\hline
\end{tabular}




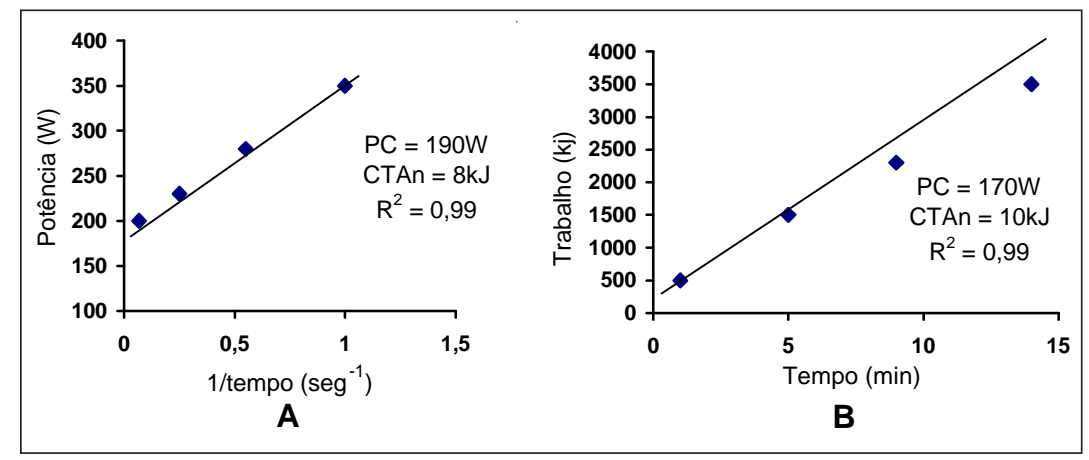

Fig. 1 - Análise de regressão e ajuste de curvas para os modelos potência-1/tempo (A) e trabalho-tempo (B) utilizados para a determinação da potência crítica $(P C)$ e da capacidade de trabalho anaeróbio (CTAn) empregando-se todas as potências. Exemplo para um sujeito.

lizadas todas as potências $\left(\mathrm{P}_{1,2,3,4}\right)$, as três maiores $\left(\mathrm{P}_{1,2,3}\right)$ e as três menores $\left(\mathrm{P}_{2,3,4}\right)$. No modelo potência-1/tempo, o yintercepto e o grau de inclinação da reta foram considerados a PC e a CTAn, respectivamente. No modelo trabalhotempo, o y-intercepto e o grau de inclinação da reta foram considerados a CTAn e a PC, respectivamente (figura 1).

\section{Análise estatística}

Os valores nas tabelas e no texto são expressos em média \pm desvio-padrão. A análise dos efeitos das cargas selecionadas e do modelo no cálculo da PC e da CTAn foi realizada pela ANOVA de dois caminhos, complementada pelo teste de Tuckey. Em todos os testes adotou-se um nível de significância de $\mathrm{P} \leq 0,05$.

\section{RESULTADOS}

Os valores médios de PC, tanto no modelo potência-1/ tempo como no trabalho-tempo, foram significantemente menores quando foram selecionadas as menores potências $\left(\mathrm{P}_{2,3,4}\right)$, do que quando se empregaram os maiores valores $\left(\mathrm{P}_{1,2,3}\right)$. Não foram encontradas diferenças, para os dois modelos, entre as maiores $\left(\mathrm{P}_{1,2,3}\right)$ e todas as potências $\left(\mathrm{P}_{1,2,3,4}\right)$. Em relação aos modelos, a $\mathrm{PC}$ determinada no modelo trabalho-tempo foi significantemente menor do que no modelo potência-1/tempo, quando se empregaram todas as potências $\left(\mathrm{P}_{1,2,3,4}\right)$ e os maiores valores $\left(\mathrm{P}_{1,2,3,4}\right)$, não existindo diferenças, entretanto, entre os menores valores de potência $\left(\mathrm{P}_{2,3,4}\right)$.

Os valores médios de CTAn, tanto no modelo potência1 /tempo como no trabalho-tempo, foram significantemente maiores quando foram selecionadas as menores potências $\left(\mathrm{P}_{2,3,4}\right)$, do que quando se empregaram os maiores valores $\left(\mathrm{P}_{1,2,3}\right)$. Não foram encontradas diferenças, para os dois modelos, entre as maiores $\left(\mathrm{P}_{1,2,3}\right)$ e todas as potências $\left(\mathrm{P}_{1,2,3,4}\right)$. Em relação aos modelos, a CTAn determinada no modelo trabalho-tempo foi significantemente maior do que
TABELA 3

Valores médios $\pm \mathrm{DP}$ da potência crítica determinada nos modelos potência-1/tempo e trabalho-tempo, utilizando todas $\left(\mathrm{P}_{1,2,3,4}\right)$ as maiores $\left(\mathrm{P}_{1,2,3}\right)$ e as menores potências $\left(P_{2,3,4}\right) \quad(N=8)$
Potência-1/tempo

(W)

$\begin{array}{lc}\mathrm{P}_{1,2,3,4} & 184,2 \pm 25,4 \\ \mathrm{P}_{1,2,3} & 190,5 \pm 23,2 \\ \mathrm{P}_{2,3,4} & 177,5 \pm 29,5^{+}\end{array}$

Trabalho-tempo

(W)
$176,4 \pm 28,8^{*}$

$183,4 \pm 22,3^{*}$

$173,9 \pm 33,3^{+}$
* $\mathrm{P}<0,05$ em relação ao modelo potência-1/tempo dentro da mesma seleção de cargas; $+\mathrm{P}<0,05$ em relação à seleção $\mathrm{P}_{1,2,3}$ dentro do mesmo modelo.

\begin{tabular}{|c|c|c|}
\hline \multicolumn{3}{|c|}{$\begin{array}{c}\text { TABELA } 4 \\
\text { Valores médios } \pm \text { DP da capacidade de trabalho } \\
\text { anaeróbio determinada nos modelos potência-1/tempo } \\
\text { e trabalho-tempo, utilizando todas }\left(\mathrm{P}_{1,2,3,4}\right) \text { as maiores } \\
\left(\mathrm{P}_{1,2,3}\right) \text { e as menores potências }\left(\mathrm{P}_{2,3,4}\right) \quad(\mathrm{N}=8)\end{array}$} \\
\hline & $\begin{array}{c}\text { Potência-1/tempo } \\
\text { (kJ) }\end{array}$ & $\begin{array}{c}\text { Trabalho-tempo } \\
\text { (kJ) }\end{array}$ \\
\hline $\mathrm{P}_{1,2,3,4}$ & $7,3 \pm 1,2$ & $9,7 \pm 2,5^{\star}$ \\
\hline $\mathrm{P}_{1,2,3}$ & $6,7 \pm 1,0$ & $8,4 \pm 1,7^{\star}$ \\
\hline$P_{2,3,4}$ & $9,7 \pm 3,9^{+}$ & $10,7 \pm 4,7^{+}$ \\
\hline
\end{tabular}

* $\mathrm{P}<0,05$ em relação ao modelo potência-1/tempo dentro da mesma seleção de cargas; $+\mathrm{P}<0,05$ em relação à seleção $\mathrm{P}_{1,2,3}$ dentro do mesmo modelo.

no modelo potência-1/tempo, quando se empregaram todas as potências $\left(\mathrm{P}_{1,2,3,4}\right)$ e os maiores valores $\left(\mathrm{P}_{1,2,3,4}\right)$, não existindo diferenças, entretanto, entre os menores valores de potência $\left(\mathrm{P}_{2,3,4}\right)$.

\section{DISCUSSÃO}

O objetivo deste estudo foi verificar o efeito da seleção das cargas e do modelo na determinação da PC e da CTAn 
durante o exercício realizado no ergômetro de braço. Concordando com estudos anteriores, que utilizaram a bicicleta ergométrica ${ }^{12-14}$, verificou-se neste estudo que tanto a seleção de cargas como o modelo utilizado interferem no cálculo da PC e da CTAn.

Housh et al. ${ }^{13}$, embora não tenham analisado especificamente esse efeito, verificaram que a PC calculada com base nas menores potências $(188 \mathrm{~W})$ e, portanto, com os maiores tempos de exaustão, foi menor do que a que utilizou as maiores potências $(216 \mathrm{~W})$. Do mesmo modo, Bishop et al. ${ }^{12}$ verificaram esse efeito quando se determinou a PC na bicicleta ergométrica utilizando as coordenadas $\left(\mathrm{W}_{\lim }-\mathrm{t}_{\lim }\right)$ das três maiores $(201 \mathrm{~W})$ e das três menores potências $(164 \mathrm{~W})$. É interessante ressaltar que, embora esse comportamento tenha ocorrido, em todos os estudos foram encontrados elevados coeficientes de linearidade $\left(\mathrm{R}^{2}=0,99-1,00\right)$ entre a $\mathrm{W}_{\text {lim }}$ e o $\mathrm{t}_{\text {lim }}$.

Bishop et al. ${ }^{12}$ propõem que essa influência da seleção das cargas na determinação da PC pode ser explicada pelo efeito da "inércia aeróbia", particularmente quando a $\mathrm{W}_{\lim }$ permite $u m t_{\text {lim }}$ abaixo de três minutos. Vanderwalle et al..$^{15}$ sugerem que, se o período no qual o metabolismo aeróbio está aumentando para próximo do máximo não é considerado, a relação $\mathrm{W}_{\text {lim }}-\mathrm{t}_{\lim }$ é modificada, resultando em maior $\mathrm{PC}$ e menor CTAn. O consumo de oxigênio em indivíduos normais aumenta no início do exercício de modo monoexponencial, atingindo uma nova fase estável após 2-3 minutos de esforço. Portanto, se a PC é um índice que deve representar a capacidade funcional aeróbia, as cargas preditivas devem permitir um $\mathrm{t}_{\text {lim }}$ de pelo menos três minutos. Em nosso estudo, apenas a maior carga utilizada permitiu duração média menor do que três minutos, o que pode explicar os maiores valores encontrados de PC quando se utilizou a combinação $\mathrm{P}_{1,2,3}$.

Em relação ao modelo de ajuste entre a $\mathrm{W}_{\lim }-\mathrm{t}_{\lim }$, Gaesser et al. ${ }^{14}$ também encontraram que o modelo potência-1/ tempo determinou maiores valores de $\mathrm{PC}(237 \mathrm{~W})$ do que o modelo trabalho-tempo $(224 \mathrm{~W})$, sendo ainda mais elevados quando se empregou o modelo potência-tempo (242W). Os autores sugerem que esse comportamento pode ser atribuído ao fato de que os modelos diferem com respeito à designação das variáveis dependentes e independentes e a unidade na qual elas são expressas, dando um peso diferente a cada coordenada $\left(\mathrm{W}_{\lim }-\mathrm{t}_{\text {lim }}\right)$ e, conseqüentemente, aos valores que podem ser encontrados para a PC e a CTAn.

Esses dados podem explicar, pelo menos em parte, as diferenças entre a PC e a intensidade correspondente ao limiar anaeróbio encontradas em alguns estudos, embora possa existir uma alta correlação entre esses índices ${ }^{7-9}$. Podem explicar, ainda, os tempos de exaustão relativamente reduzidos (10-20min) quando os indivíduos se exercitaram na $\mathrm{PC}^{9-11}$, embora o conceito deste índice pressuponha uma intensidade que pode ser sustentada por um longo período de tempo sem exaustão.

Com base nesses dados, pode-se concluir que a seleção das cargas preditivas e o modelo de ajuste da relação $\mathrm{W}_{\text {lim}}-$ $\mathrm{t}_{\text {lim }}$ interferem no cálculo da PC e da CTAn durante o exercício realizado no ergômetro de braço, mostrando que a massa muscular participante no exercício parece não modificar esse comportamento, tendo em vista que esse fenômeno já havia sido descrito para a bicicleta ergométrica ${ }^{12} \mathrm{e}$ a corrida ${ }^{16}$. Portanto, deve-se ter especial atenção na escolha das cargas que serão empregadas na determinação da PC, especialmente para a utilização deste índice na prescrição da intensidade do treinamento aeróbio.

\section{REFERÊNCIAS}

1. Monod H, Scherrer J. The work capacity of a synergic muscular group. Ergonomics 1965;8:329-38.

2. Moritani TA, Nagata HA, deVries HA, Muro M. Critical power as a measure of physical work capacity and anaerobic threshold. Ergonomics 1981;24:339-50.

3. Guglielmo LGA, Denadai BS. Avaliação da capacidade anaeróbia de nadadores utilizando o conceito de potência crítica. Anais do Simpósio Internacional de Ciências do Esporte, São Paulo-SP, 1998;73.

4. Guglielmo LGA, Denadai BS. Correlação do teste de Wingate de braço com a capacidade de trabalho anaeróbio determinada através do conceito de velocidade crítica na natação. Motriz 1999;5:92.

5. Denadai BS, Greco CC, Donega MR. Comparação entre a velocidade de limiar anaeróbio e a velocidade crítica em nadadores com idade de 10 a 15 anos. Rev Paul Educ Fís 1997;1 1:128-33.

6. Caputo F, de Lucas RD, Denadai BS. Correlação entre velocidade crítica e o índice de braçada nas distâncias de 50, 100, 200 e 400 metros no estilo crawl. Motriz 1999;5:90.

7. Wakayoshi K, Yoshida T, Udo M, Kasai T, Moritani T, Mutoh Y, Miyashita M. A simple method for determining critical speed as swimming fatigue threshold in competitive swimming. Int J Sports Med 1992;13:367-71.

8. Housh TJ, deVries HA, Tichy MW, Smyth KD. The relationship between critical power and the onset of blood lactate accumulation. J Sports Med Phys Fitness 1991;31:31-6.

9. McLellan TM, Cheung KSY. A comparative evaluation of the individual anaerobic threshold and the critical power. Med Sci Sports Exerc 1992;24:543-50.

10. Jenkins D G, Quigley B M. Blood lactate in trained cyclist during cycle ergometry at critical power. Eur J Appl Physiol 1990;61:278-83.

11. de Lucas RD, Caputo F, Denadai BS. Tempo de exaustão na velocidade crítica na natação. Motriz 1999;5:90.

12. Bishop D, Jenkins DG, Howard A. The critical power is dependent on the duration of the predictive exercise tests chosen. Int J Sports Med 1998;19:125-9.

13. Housh DJ, Housh TJ, Bauge SM. A methodological consideration for determination of critical power and anaerobic work capacity. Res Q Exerc Sport 1990;61:406-9.

14. Gaesser GA, Carnevale TJ, Garfinkel A, Walter DO, Womack CJ. Estimation of critical power with nonlinear and linear models. Med Sci Sports Exerc 1995;27:1430-8.

15. Vanderwalle H, Kapitaniak b, Grun S, Raveneau S, Monod H. Comparison between a $30-\mathrm{s}-$ all-out test and a time-work test on a cycle ergometer. Eur J Appl Physiol 1989;58:375-81.

16. Hopkins WG, Edmund IM, Hamilton BH, MacFarlane DJ, Ross BH. Relation between power and endurance for treadmill running of short duration. Ergonomics 1989;32:1565-71. 